\title{
Publisher Correction: Long-distance spin transport through a graphene quantum Hall antiferromagnet
}

Petr Stepanov, Shi Che, Dmitry Shcherbakov, Jiawei Yang, Ruoyu Chen, Kevin Thilahar, Greyson Voigt, Marc W. Bockrath, Dmitry Smirnov, Kenji Watanabe (D), Takashi Taniguchi, Roger K. Lake (D), Yafis Barlas (D), Allan H. MacDonald (D) and

Chun Ning Lau (i)

Correction to: Nature Physics https://doi.org/10.1038/s41567-018-0161-5, published online 11 June 2018.

In the version of this Letter originally published, the number in the middle yellow box of Fig. $2 \mathrm{~d}$ was incorrectly given as +2 ; it should have been 0 . This has now been corrected.

\section{Author Correction: Pair-breaking quantum phase transition in superconducting nanowires}

Hyunjeong Kim, Frédéric Gay, Adrian Del Maestro (D), Benjamin Sacépé (D) and Andrey Rogachev (D)

Correction to: Nature Physics https://doi.org/10.1038/s41567-018-0179-8, published online 9 July 2018.

In the version of this Letter originally published, one of the authors' first names was incorrectly spelled Fédéric; it should have read Frédéric. This has now been corrected.

\section{Publisher Correction: Maximal spontaneous photon emission and energy loss from free electrons}

Yi Yang (D), Aviram Massuda, Charles Roques-Carmes (D), Steven E. Kooi (D), Thomas Christensen, Steven G. Johnson, John D. Joannopoulos, Owen D. Miller (D), Ido Kaminer (D) and Marin Soljačić

Correction to: Nature Physics https://doi.org/10.1038/s41567-018-0180-2, published online 16 July 2018.

In the version of this Letter originally published, an older version of the Supplementary Information was uploaded by mistake, in which the notation did not match the main text. This has been corrected.

Published online: 23 July 2018

https://doi.org/10.1038/s41567-018-0252-3

\section{Publisher Correction: Entropic effects in cell lineage tree packings}

Jasmin Imran Alsous, Paul Villoutreix, Norbert Stoop, Stanislav Y. Shvartsman and Jörn Dunkel (iD

Correction to: Nature Physics https://doi.org/10.1038/s41567-018-0202-0, published online 16 July 2018.

In the version of this Letter originally published, the citations to equation (1) in Fig. 3 caption and the main text were incorrect; they should have been to equation (2). This has now been corrected. 